Supporting Information

For

\title{
Characterization of glycosphingolipids and their diverse lipid forms through two-stage spectral matching of LC-MS/MS data
}

\author{
Laura S. Bailey, ${ }^{\dagger}$ Fanran Huang, ${ }^{\dagger}$ Tianqi Gao,${ }^{\dagger}$ Jinying Zhao,${ }^{\ddagger}$ Kari B. Basso, ${ }^{\dagger}$ and \\ Zhongwu Guo ${ }^{\dagger, *}$ \\ †Department of Chemistry and "Department of Epidemiology, University of Florida, \\ Gainesville, Florida 32611, USA \\ *Corresponding author E-mail: zguo@chem.ufl.edu
}

\section{Table of Contents}

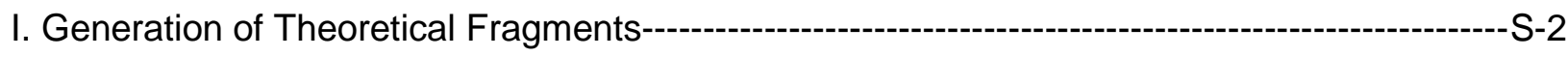

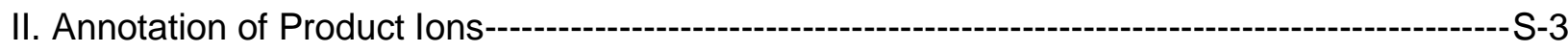

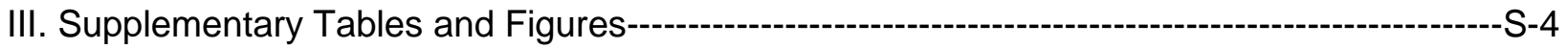




\section{Generation of Theoretical Fragments}

A list of all theoretical carbohydrate fragment ions was generated using GlycanWorkbench for the reference GSLs [GM3 (36:1), GM2 (36:1), and GD2 (36:1)], as well as for the deuterated GM3- $d_{5}(36: 1)$. The reducing end was modified with a user-defined ceramide (C36:1) aglycon (see below) having a mass of 547.5328 for the reference GSLs and 552.5643 for the deuterated GM3. B, C, Y, Z-type fragments [i.e., $B(1-n), C(1-n), Y(1-n-1)$, and $Z(1-n-1)$ ] and internal fragments (e.g., $B Y, Y Y$, etc.) were generated for protonated ions, allowing for a maximum of 2 cleavages. Internal carbohydrate fragments are formed upon cleavage of at least two glycosidic bonds, with the charge remaining on a carbohydrate fragment that is not the non-reducing end gragment. This is common for complex and/or branched glycans. Actually, the more branches a glycan has, the more internal carbohydrate fragments it can generate via the cleavage of two or more glycosidic bonds. For the complex GSL, GD2, a maximum of 3 cleavages were allowed. $\mathrm{YO}$ and $\mathrm{ZO}$ fragments manually calculated with the assistance of ChemDraw.

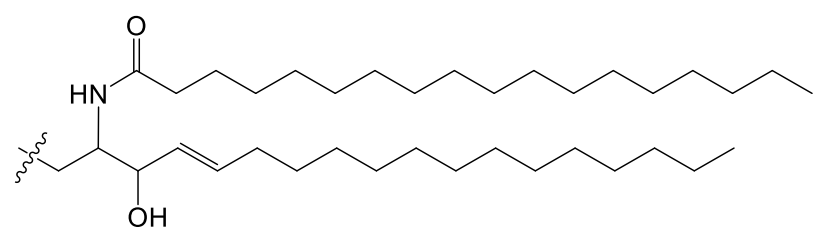

Chemical Formula: $\mathrm{C}_{36} \mathrm{H}_{69} \mathrm{NO}_{2}$; Exact Mass: 547.5328

Ceramide fragments (e.g., N", U, V, etc.) were generated using the in-silico glycosylceramide fragment predictor from Lipid Maps (https://www.lipidmaps.org/tools/ms/SP insilico form.php) for an acyl 18:0 and sphingosine d18:1 lipid definition. For example, the N" ion has the following structure:

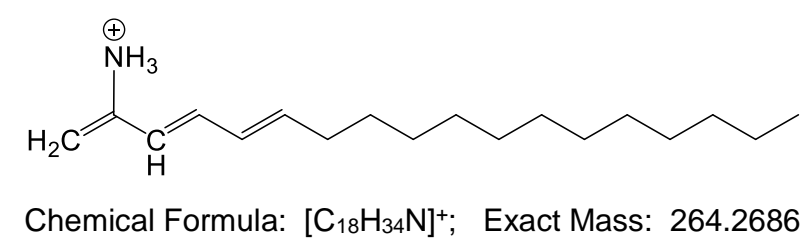

Water losses were manually calculated for all fragments $(B / C, Y / Z$, internal fragments, and $M H)$ by reducing the ion mass by $\mathrm{m} / \mathrm{z} 18.0106$. Additional lipid form masses were generated by modifying the glycolipid fragment masses by $\mathrm{m} / \mathrm{z} 14.0157$ (for $-\mathrm{CH}_{2}$ - addition or loss), $\mathrm{m} / \mathrm{z} 28.0313$ (for $-\mathrm{C}_{2} \mathrm{H}_{4}$ - addition or loss), and/or $\mathrm{m} / \mathrm{z} 2.0165$ (for $2 \mathrm{H}$ addition or loss). The mass modification required was determined by the precursor mass difference between the other lipid form and reference.

Matching was performed using an in-house Excel program originally written by Dr. Matt Bell. A matching threshold of $20 \mathrm{ppm}$ was used for the program. All fragment ions were manually validated. In the rare likelihood that multiple product ions could be assigned the same fragment name, several criteria were developed to determine the most likely candidate for annotation. Annotations were assigned to product ions yielding the highest mass accuracy (i.e., lowest ppm). In some cases where mass 
accuracies were similar, the highest ion abundance was generally chosen, although mass similarity to other lipid forms and/or matching with isomer ion masses (within the product ion's expected isotopic envelope) was used to determine the more likely product ion assignment.

The chemical structures of GM3 (36:1), GM2 (36:1), and GD2 (36:1) are presented below:
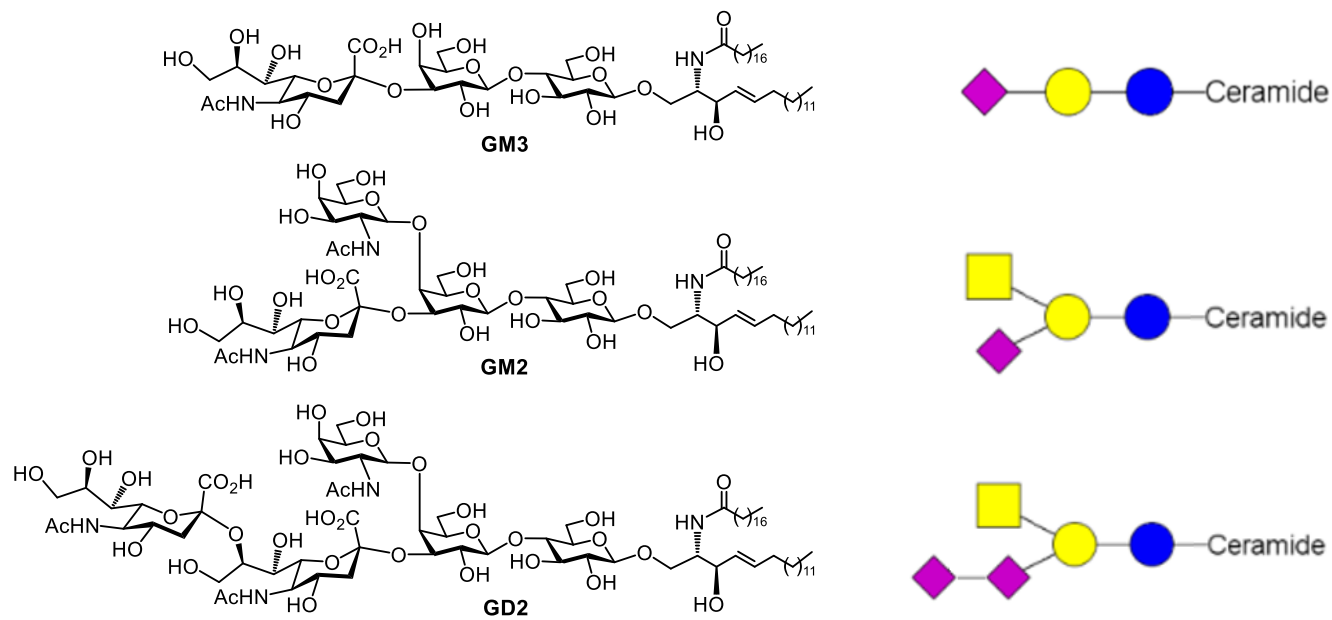

\section{Annotation of Product lons}

Due to many possible isobaric fragments (i.e., fragments having identical or similar masses but different structures and, therefore, different names), GSL fragment ions were named according to the following rules.

First, B, C, Y, Z, and internal fragment (i.e., BY, CY, BZ, CZ, YY, ZZ, YZ, ZY) annotations were given high priority. Water loss was annotated only if no other "pure" glycan type nomenclature could be applied. For example, no C-type (or internal fragments initiated through C-type cleavages) dehydration ions were named as these ions could also be assigned to B-type (or internal fragments initiated through B-type cleavages) fragmentation. Similarly, Y-type (or internal fragments initiated through Y-type cleavages) dehydration ions were not assigned as these are isobaric with Z-type annotations.

Second, B-type and Y-type annotations, including fragments initiated through B-type or Y-type cleavages (e.g., BY or YZ), were given priority over C-type and Z-type (or internal fragments initiated by these cleavage types) annotations. For example, if a $B$ ion was isobaric with a $C Y$ ion, the product ion was annotated as a $B$ fragment. Similarly, if a $Y$ ion was isobaric with a $Z Y$ ion, the product ion was annotated as a $Y$ fragment.

Third, and related to the previous rule, isobaric internal fragment names were prioritized for which B-type or Y-type cleavages initiated the dissociation event. For example, every $Y Z$ ion could have also been annotated $Z Y$; however, all such product ions are annotated as $Y Z$ following the above rule that $Y$ type annotations are given priority over Z-type annotations. 


\section{Supplementary Tables and Figures}

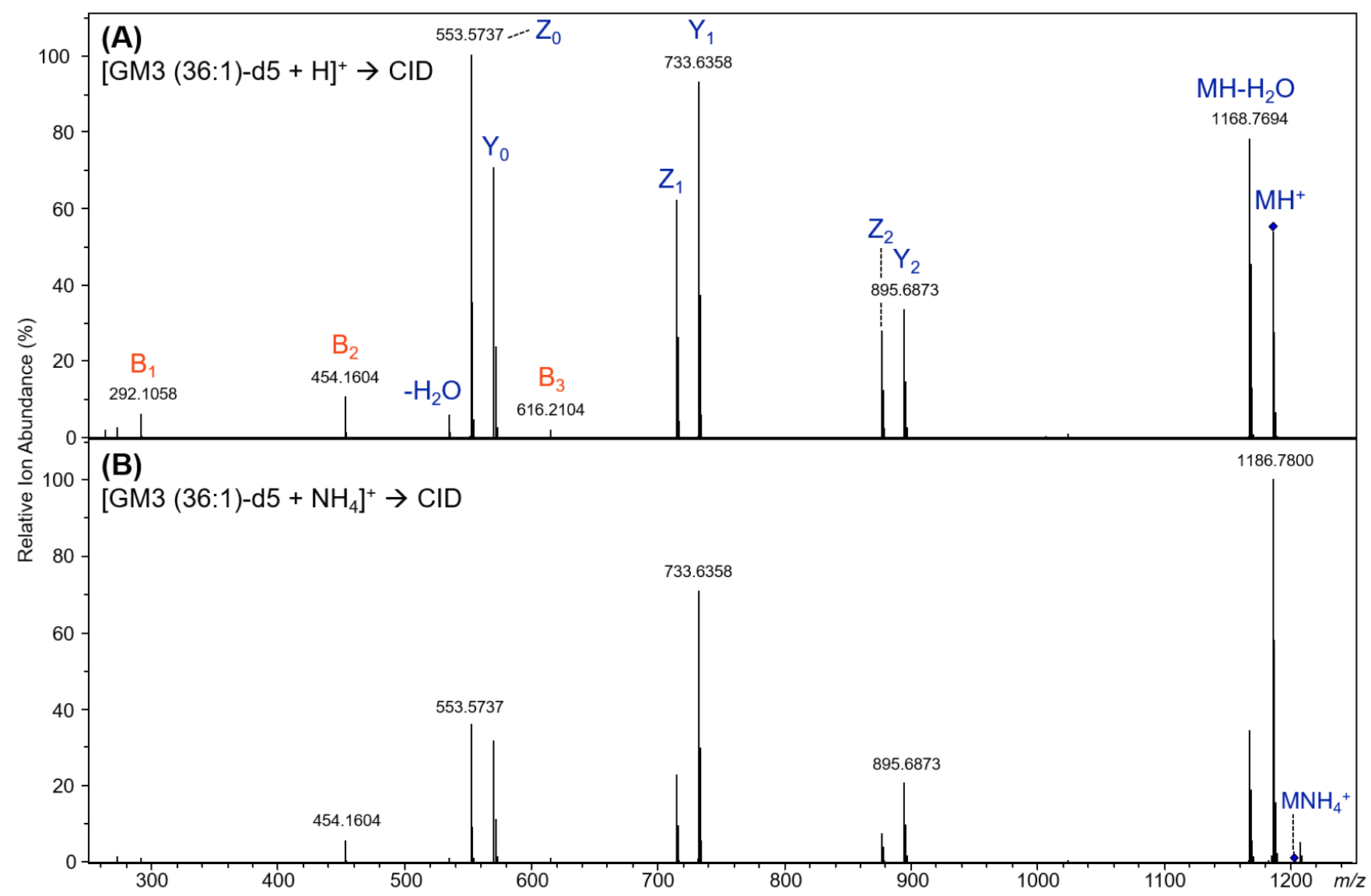

Figure S1: Product spectra for the protonated (A) and ammoniated (B) GM3 (36:1)-d5 ions. The precursor ions in both spectra are labeled with the blue diamond. The product ion designations are labeled in (A). 
Table S1: All matched product ion masses and fragment assignments for the GM3 lipid forms presented in Figure 2 (left to right): GM3 (36:1), GM3 (38:1), GM3 (40:1), and GM3-d $d_{5}(36: 1)$. For each lipid form, the observed mass $(\mathrm{m} / \mathrm{z})$, relative ion intensity $(1 \%)$, and mass accuracy (compared to the theoretically calculated ion mass) (ppm) are provided. The fragment masses have been divided into carbohydrate fragments (top), which includes any product ion assignment that does not change mass with lipid form, and glycolipid fragments (bottom), which includes any product ion assignment that shifts mass as lipid form changes, in order of increasing mass.

\begin{tabular}{|c|c|c|c|c|c|c|c|c|c|c|c|c|}
\hline \multirow{2}{*}{$\begin{array}{l}\text { Fragment } \\
\text { Assignment }\end{array}$} & \multicolumn{3}{|c|}{ GM3 (36:1) } & \multicolumn{3}{|c|}{ GM3 (38:1) } & \multicolumn{3}{|c|}{ GM3 (40:1) } & \multicolumn{3}{|c|}{ GM3-d $5(36: 1)$} \\
\hline & $m / z$ & $1 \%$ & ppm & $m / z$ & $1 \%$ & ppm & $m / z$ & $1 \%$ & ppm & $m / z$ & $1 \%$ & ppm \\
\hline \multicolumn{13}{|c|}{ Carbohydrate fragments } \\
\hline $\mathrm{B} 1-\mathrm{H}_{2} \mathrm{O}$ & 274.0949 & 2 & 10.1 & 274.0956 & 3 & 12.7 & 274.0958 & 3 & 13.4 & 274.0957 & 3 & 13.1 \\
\hline B1 & 292.1064 & 7 & 12.7 & 292.1063 & 7 & 12.3 & 292.1067 & 6 & 13.7 & 292.1065 & 7 & 13.0 \\
\hline $\mathrm{C} 1$ & & & & & & & 310.1176 & 0.1 & 14.0 & 310.1161 & 0.1 & 9.2 \\
\hline B3Y2 & & & & & & & & & & 325.1155 & 0.1 & 8.0 \\
\hline $\mathrm{B} 2-\mathrm{H}_{2} \mathrm{O}$ & & & & & & & 436.1460 & 0.1 & 2.4 & 436.1469 & 0.1 & 4.5 \\
\hline B2 & 454.1597 & 10 & 9.2 & 454.1587 & 11 & 7.0 & 454.1589 & 11 & 7.5 & 454.1587 & 11 & 7.0 \\
\hline $\mathrm{C} 2$ & & & & 472.1708 & 0.2 & 10.0 & 472.1714 & 0.1 & 11.3 & 472.1678 & 0.2 & 3.6 \\
\hline B3- $\mathrm{H}_{2} \mathrm{O}$ & & & & & & & 598.2014 & 0.1 & 6.1 & 598.1991 & 0.1 & 2.3 \\
\hline B3 & 616.2099 & 1 & 2.6 & 616.2102 & 2 & 3.1 & 616.2115 & 2 & 5.2 & 616.2104 & 2 & 3.4 \\
\hline \multicolumn{13}{|c|}{ Glycolipid fragments } \\
\hline N" & 264.2726 & 2 & 15.3 & 264.2695 & 0.2 & 3.5 & 264.2725 & 0.8 & 14.9 & 264.2720 & 2 & 13.0 \\
\hline $\mathrm{ZO}-\mathrm{H}_{2} \mathrm{O}$ & 530.5345 & 5 & 9.4 & 558.5628 & 6 & 3.5 & 586.5947 & 6 & 4.4 & 535.5637 & 6 & 5.2 \\
\hline Z0 & 548.5426 & 100 & 4.6 & 576.5735 & 100 & 3.6 & 604.6051 & 99 & 4.0 & 553.5737 & 100 & 4.0 \\
\hline Y0 & 566.5533 & 63 & 4.6 & 594.5838 & 77 & 3.0 & 622.6155 & 77 & 3.5 & 571.5842 & 71 & 3.7 \\
\hline $\mathrm{Z} 1-\mathrm{H}_{2} \mathrm{O}$ & & & & 720.6190 & 0.3 & 7.3 & 748.6464 & 0.3 & 1.8 & & & \\
\hline $\mathrm{Z1}$ & 710.5945 & 52 & 2.1 & 738.6252 & 61 & 1.2 & 766.6569 & 62 & 1.7 & 715.6255 & 62 & 1.5 \\
\hline Y1 & 728.6047 & 76 & 1.6 & 756.6354 & 98 & 0.7 & 784.6672 & 100 & 1.3 & 733.6358 & 93 & 1.1 \\
\hline Z2 & 872.6452 & 19 & -0.7 & 900.6767 & 25 & -0.5 & 928.7085 & 26 & 0.1 & 877.6766 & 28 & -0.7 \\
\hline Y2 & 890.6560 & 22 & -0.4 & 918.6873 & 33 & -0.4 & 946.7187 & 36 & -0.3 & 895.6873 & 34 & -0.5 \\
\hline $\mathrm{MH}-\mathrm{H}_{2} \mathrm{O}$ & 1163.7383 & 51 & -2.5 & 1191.7691 & 75 & -2.9 & 1219.8016 & 78 & -1.8 & 1168.7694 & 78 & -2.8 \\
\hline $\mathrm{MH}$ & 1181.7486 & 29 & -2.7 & 1209.7793 & 60 & -3.1 & 1237.8117 & 71 & -2.2 & 1186.7802 & 54 & -2.5 \\
\hline
\end{tabular}


Table S2: Table S1 data of product ion masses and fragment assignments for GM3 lipid forms GM3 (36:1), GM3 (38:1), GM3 (40:1), and GM3- $d_{5}(36: 1)$ presented according to the relative ion intensity (I\%) from the highest to the lowest for the carbohydrate (top) and glycolipid fragments, respectively. The dashed line represents a 1\% threshold (according to the reference, GM3 (36:1)), below which fragment matching is not always guaranteed.

\begin{tabular}{|c|c|c|c|c|c|c|c|c|}
\hline \multirow{2}{*}{$\begin{array}{l}\text { Fragment } \\
\text { Assignment }\end{array}$} & \multicolumn{2}{|c|}{ GM3 (36:1) } & \multicolumn{2}{|c|}{ GM3 (38:1) } & \multicolumn{2}{|c|}{ GM3 (40:1) } & \multicolumn{2}{|c|}{ GM3-d $5(36: 1)$} \\
\hline & $m / z$ & $1 \%$ & $m / z$ & $1 \%$ & $m / z$ & $1 \%$ & $m / z$ & $1 \%$ \\
\hline \multicolumn{9}{|c|}{ Carbohydrate fragments } \\
\hline B2 & 454.1597 & 10 & 454.1587 & 11 & 454.1589 & 11 & 454.1587 & 11 \\
\hline B1 & 292.1064 & 7 & 292.1063 & 7 & 292.1067 & 6 & 292.1065 & 7 \\
\hline $\mathrm{B} 1-\mathrm{H}_{2} \mathrm{O}$ & 274.0949 & 2 & 274.0956 & 3 & 274.0958 & 3 & 274.0957 & 3 \\
\hline B3 & 616.2099 & 1 & 616.2102 & 2 & 616.2115 & 2 & 616.2104 & 2 \\
\hline C1 & & & & & 310.1176 & 0.1 & 310.1161 & 0.1 \\
\hline B3Y2 & & & & & & & 325.1155 & 0.1 \\
\hline $\mathrm{B} 2-\mathrm{H}_{2} \mathrm{O}$ & & & & & 436.1460 & 0.1 & 436.1469 & 0.1 \\
\hline $\mathrm{C} 2$ & & & 472.1708 & 0.2 & 472.1714 & 0.1 & 472.1678 & 0.2 \\
\hline B3- $\mathrm{H}_{2} \mathrm{O}$ & & & & & 598.2014 & 0.1 & 598.1991 & 0.1 \\
\hline \multicolumn{9}{|c|}{ Glycolipid fragments } \\
\hline $\mathrm{ZO}$ & 548.5426 & 100 & 576.5735 & 100 & 604.6051 & 99 & 553.5737 & 100 \\
\hline Y1 & 728.6047 & 76 & 756.6354 & 98 & 784.6672 & 100 & 733.6358 & 93 \\
\hline Y0 & 566.5533 & 63 & 594.5838 & 77 & 622.6155 & 77 & 571.5842 & 71 \\
\hline Z1 & 710.5945 & 52 & 738.6252 & 61 & 766.6569 & 62 & 715.6255 & 62 \\
\hline $\mathrm{MH}-\mathrm{H}_{2} \mathrm{O}$ & 1163.7383 & 51 & 1191.7691 & 75 & 1219.8016 & 78 & 1168.7694 & 78 \\
\hline Y2 & 890.6560 & 22 & 918.6873 & 33 & 946.7187 & 36 & 895.6873 & 34 \\
\hline Z2 & 872.6452 & 19 & 900.6767 & 25 & 928.7085 & 26 & 877.6766 & 28 \\
\hline $\mathrm{ZO}-\mathrm{H}_{2} \mathrm{O}$ & 530.5345 & 5 & 558.5628 & 6 & 586.5947 & 6 & 535.5637 & 6 \\
\hline $\mathrm{Z} 1-\mathrm{H}_{2} \mathrm{O}$ & & & 720.6190 & 0.3 & 748.6464 & 0.3 & & \\
\hline
\end{tabular}


Table S3: Product ion masses and fragment assignments for the GM2 lipid forms presented in Figure 4 (left to right): GM2 (36:1), GM2 (38:1), and GM2 (40:1). For each lipid form, the observed mass $(\mathrm{m} / \mathrm{z})$, relative ion intensity $(\mathrm{l} \%)$, and mass accuracy (compared to the theoretically calculated ion mass) (ppm) are provided. The fragment masses have been divided into carbohydrate (top) and glycolipid fragments in order of increasing mass.

\begin{tabular}{|c|c|c|c|c|c|c|c|c|c|}
\hline \multirow{2}{*}{$\begin{array}{l}\text { Fragment } \\
\text { Assignment }\end{array}$} & \multicolumn{3}{|c|}{ GM2 (36:1) } & \multicolumn{3}{|c|}{ GM2 (38:1) } & \multicolumn{3}{|c|}{ GM2 (40:1) } \\
\hline & $m / z$ & $1 \%$ & ppm & $m / z$ & $1 \%$ & ppm & $m / z$ & $1 \%$ & ppm \\
\hline \multicolumn{10}{|c|}{ Carbohydrate fragments } \\
\hline $\mathrm{B} 1 \mathrm{a}-\mathrm{H}_{2} \mathrm{O}$ & 274.0953 & 0.7 & 11.6 & 274.0963 & 0.6 & 15.2 & 274.0927 & 0.6 & 2.1 \\
\hline B1a & 292.1061 & 2 & 11.7 & 292.1062 & 1 & 12.0 & 292.1050 & 2 & 7.9 \\
\hline $\mathrm{C} 1 \mathrm{a}$ & 310.1165 & 0.2 & 10.5 & 310.1165 & 0.2 & 10.5 & 310.1150 & 0.5 & 5.6 \\
\hline B2Y2a & 366.1427 & 14 & 8.8 & 366.1429 & 12 & 9.4 & 366.1423 & 16 & 7.7 \\
\hline C2Y2a & 384.1530 & 0.2 & 7.7 & 384.1531 & 0.2 & 8.0 & 384.1515 & 0.2 & 3.8 \\
\hline $\mathrm{B} 2 \mathrm{Z} 2 \mathrm{~b}-\mathrm{H}_{2} \mathrm{O}$ & 418.1360 & 0.1 & 3.9 & & & & & & \\
\hline $\mathrm{B} 2 \mathrm{Z} 2 \mathrm{~b}$ & 436.1482 & 0.2 & 7.4 & 436.1467 & 0.2 & 4.0 & & & \\
\hline B2Y2b & 454.1583 & 4 & 6.1 & 454.1581 & 4 & 5.7 & 454.1575 & 3 & 4.4 \\
\hline B3Y2a & 528.1941 & 10 & 3.4 & 528.1949 & 9 & 4.9 & 528.1951 & 12 & 5.3 \\
\hline B3Y2b & 616.2108 & 1 & 4.1 & 616.2100 & 1 & 2.8 & 616.2113 & 1 & 4.9 \\
\hline B2 & 657.2360 & 14 & 1.7 & 657.2366 & 12 & 2.6 & 657.2368 & 13 & 2.9 \\
\hline C2 & 675.2461 & 2 & 1.0 & 675.2479 & 2 & 3.6 & 675.2488 & 2 & 5.0 \\
\hline B3- $\mathrm{H}_{2} \mathrm{O}$ & 801.2755 & 0.2 & -2.0 & 801.2749 & 0.2 & -2.8 & 801.2744 & 0.3 & -3.4 \\
\hline B3 & 819.2872 & 73 & -0.6 & 819.2879 & 71 & 0.2 & 819.2872 & 84 & -0.6 \\
\hline \multicolumn{10}{|c|}{ Glycolipid fragments } \\
\hline N" & 264.2729 & 0.2 & 16.4 & & & & & & \\
\hline $\mathrm{ZO}-\mathrm{H}_{2} \mathrm{O}$ & 530.5319 & 0.5 & 4.5 & 558.5636 & 0.3 & 5.0 & 586.5904 & 0.5 & -2.9 \\
\hline Z0 & 548.5418 & 8 & 3.1 & 576.5735 & 7 & 3.6 & 604.6035 & 8 & 1.3 \\
\hline Y0 & 566.5527 & 6 & 3.5 & 594.5838 & 6 & 3.0 & 622.6163 & 8 & 4.8 \\
\hline Z1 & 710.5932 & 4 & 0.3 & 738.6244 & 4 & 0.1 & 766.6565 & 4 & 1.2 \\
\hline Y1 & 728.6039 & 13 & 0.5 & 756.6356 & 12 & 1.0 & 784.6691 & 13 & 3.7 \\
\hline Y2bZ2a & 872.6441 & 2 & -2.0 & 900.6766 & 1 & -0.6 & 928.7016 & 2 & -7.3 \\
\hline Y2aY2b & 890.6550 & 4 & -1.6 & 918.6866 & 4 & -1.2 & 946.7188 & 4 & -0.2 \\
\hline Z2a & 1075.7217 & 2 & -3.2 & 1103.7534 & 1 & -2.8 & 1131.7900 & 1 & 1.9 \\
\hline Y2a & 1093.7319 & 2 & -3.5 & 1121.7642 & 2 & -2.6 & 1149.7920 & 1 & -5.5 \\
\hline Z2b & 1163.7372 & 6 & -3.5 & 1191.7691 & 5 & -2.9 & 1219.8008 & 5 & -2.5 \\
\hline Y2b & 1181.7473 & 31 & -3.8 & 1209.7794 & 30 & -3.1 & 1237.8092 & 24 & -4.2 \\
\hline $\mathrm{MH}-\mathrm{H}_{2} \mathrm{O}$ & 1366.8139 & 50 & -4.9 & 1394.8463 & 45 & -4.0 & 1422.8768 & 38 & -4.5 \\
\hline $\mathrm{MH}$ & 1384.8243 & 100 & -5.0 & 1412.8567 & 100 & -4.1 & 1440.8867 & 100 & -4.9 \\
\hline
\end{tabular}


Table S4: Table S3 data of product ion masses and fragment assignments for GM2 lipid forms GM2 (36:1), GM2 (38:1), and GM2 (40:1) presented according to the relative ion intensity (I\%) from the highest to the lowest for the carbohydrate (top) and glycolipid fragments, respectively. The dashed line represents a 1\% threshold (according to the reference, GM2 (36:1)), below which fragment matching is not always guaranteed.

\begin{tabular}{|c|c|c|c|c|c|c|}
\hline \multirow{2}{*}{$\begin{array}{l}\text { Fragment } \\
\text { Assignment }\end{array}$} & \multicolumn{2}{|c|}{ GM2 (36:1) } & \multicolumn{2}{|c|}{ GM2 (38:1) } & \multicolumn{2}{|c|}{ GM2 (40:1) } \\
\hline & $m / z$ & $1 \%$ & $m / z$ & $1 \%$ & $m / z$ & $1 \%$ \\
\hline \multicolumn{7}{|c|}{ Carbohydrate fragments } \\
\hline B3 & 819.2872 & 73 & 819.2879 & 71 & 819.2872 & 84 \\
\hline $\mathrm{B} 2 \mathrm{Y} 2 \mathrm{a}$ & 366.1427 & 14 & 366.1429 & 12 & 366.1423 & 16 \\
\hline B2 & 657.2360 & 14 & 657.2366 & 12 & 657.2368 & 13 \\
\hline B3Y2a & 528.1941 & 10 & 528.1949 & 9 & 528.1951 & 12 \\
\hline B2Y2b & 454.1583 & 4 & 454.1581 & 4 & 454.1575 & 3 \\
\hline B1a & 292.1061 & 2 & 292.1062 & 1 & 292.1050 & 2 \\
\hline $\mathrm{C} 2$ & 675.2461 & 2 & 675.2479 & 2 & 675.2488 & 2 \\
\hline B3Y2b & 616.2108 & 1 & 616.2100 & 1 & 616.2113 & 1 \\
\hline $\mathrm{B} 1 \mathrm{a}-\mathrm{H}_{2} \mathrm{O}$ & 274.0953 & 0.7 & 274.0963 & 0.6 & 274.0927 & 0.6 \\
\hline $\mathrm{C} 1 \mathrm{a}$ & 310.1165 & 0.2 & 310.1165 & 0.2 & 310.1150 & 0.5 \\
\hline C2Y2a & 384.1530 & 0.2 & 384.1531 & 0.2 & 384.1515 & 0.2 \\
\hline B2Z2b & 436.1482 & 0.2 & 436.1467 & 0.2 & & \\
\hline $\mathrm{B} 3-\mathrm{H}_{2} \mathrm{O}$ & 801.2755 & 0.2 & 801.2749 & 0.2 & 801.2744 & 0.3 \\
\hline $\mathrm{B} 2 \mathrm{Z} 2 \mathrm{~b}-\mathrm{H}_{2} \mathrm{O}$ & 418.1360 & 0.1 & & & & \\
\hline \multicolumn{7}{|c|}{ Glycolipid fragments } \\
\hline $\mathrm{MH}-\mathrm{H}_{2} \mathrm{O}$ & 1366.8139 & 50 & 1394.8463 & 45 & 1422.8768 & 38 \\
\hline Y2b & 1181.7473 & 31 & 1209.7794 & 30 & 1237.8092 & 24 \\
\hline Y1 & 728.6039 & 13 & 756.6356 & 12 & 784.6691 & 13 \\
\hline Z0 & 548.5418 & 8 & 576.5735 & 7 & 604.6035 & 8 \\
\hline YO & 566.5527 & 6 & 594.5838 & 6 & 622.6163 & 8 \\
\hline Z2b & 1163.7372 & 6 & 1191.7691 & 5 & 1219.8008 & 5 \\
\hline Y2aY2b & 890.6550 & 4 & 918.6866 & 4 & 946.7188 & 4 \\
\hline Z1 & 710.5932 & 4 & 738.6244 & 4 & 766.6565 & 4 \\
\hline Y2bZ2a & 872.6441 & 2 & 900.6766 & 1 & 928.7016 & 2 \\
\hline Z2a & 1075.7217 & 2 & 1103.7534 & 1 & 1131.7900 & 1 \\
\hline Y2a & 1093.7319 & 2 & 1121.7642 & 2 & 1149.7920 & 1 \\
\hline $\mathrm{ZO}-\mathrm{H}_{2} \mathrm{O}$ & 530.5319 & 0.5 & 558.5636 & 0.3 & 586.5904 & 0.5 \\
\hline
\end{tabular}




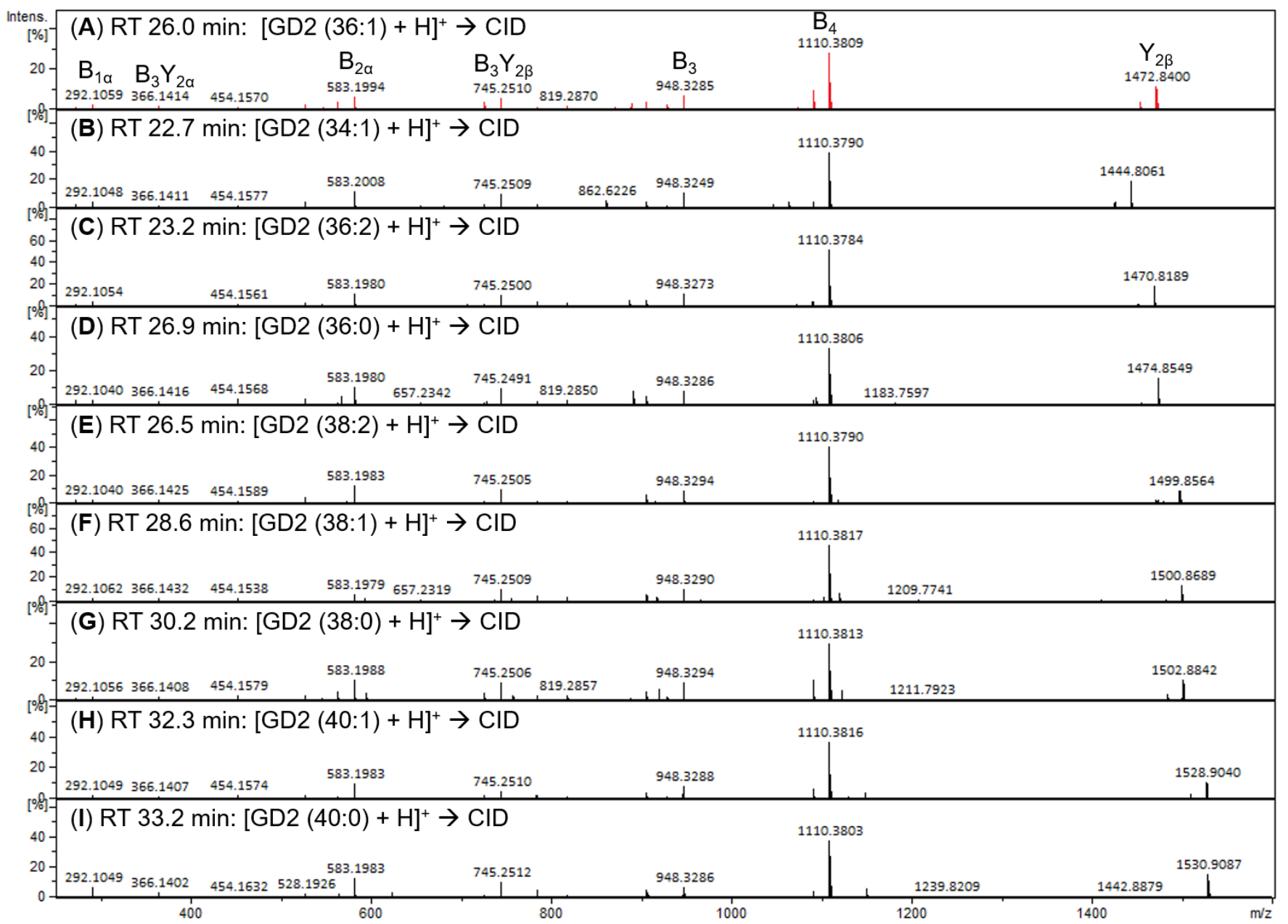

Figure S2: Product spectra of reference GD2 (36:1) (A, red) and the observed lipid forms detected through two-stage searching of the LC-MS/MS data shown in Figure 7C (top to bottom): (B) GD2 (34:1), (C) GD2 (36:0), (D) GD2 (36:2), (E) GD2 (38:0), (F) GD2 (38:1), (G) GD2 (38:2), (H) GD2 (40:1), and (I) GD2 (40:2). Some of the more abundant GD2 species product ions are labeled in (A). 
Table S5: Product ion masses and fragment assignments for the GD2 lipid forms presented in Figure 6 and Figure S1. Due to the number of lipid forms detected, only the lipid forms with 34 and 36 carbons are given here (left to right): GD2 (36:1) (reference product ion list), GD2 (34:1), GD2 (36:0), and GD2 (36:2). For each lipid form, observed mass $(\mathrm{m} / \mathrm{z})$, relative ion intensity $(1 \%)$, and mass accuracy (compared to the theoretically calculated ion mass) (ppm) are provided. The fragment masses have been divided into carbohydrate (top) and glycolipid fragments in order of increasing mass.

\begin{tabular}{|c|c|c|c|c|c|c|c|c|c|c|c|c|}
\hline \multirow{2}{*}{$\begin{array}{l}\text { Fragment } \\
\text { Assignment }\end{array}$} & \multicolumn{3}{|c|}{ GD2 (36:1) } & \multicolumn{3}{|c|}{ GD2 (34:1) } & \multicolumn{3}{|c|}{ GD2 (36:2) } & \multicolumn{3}{|c|}{ GD2 (36:0) } \\
\hline & $m / z$ & $1 \%$ & ppm & $m / z$ & $1 \%$ & ppm & $m / z$ & $1 \%$ & ppm & $m / z$ & $1 \%$ & ppm \\
\hline \multicolumn{13}{|c|}{ Carbohydrate fragments } \\
\hline B2aZ3a & 274.0950 & 2 & 10.5 & 274.0934 & 3 & 4.6 & 274.0930 & 3 & 3.2 & 274.0946 & 3 & 9.0 \\
\hline B1a & 292.1059 & 3 & 11.0 & 292.1048 & 5 & 7.2 & 292.1054 & 6 & 9.3 & 292.1040 & 3 & 4.5 \\
\hline C1a & & & & 310.1143 & 0.3 & 3.4 & & & & 310.1162 & 0.2 & 9.5 \\
\hline B3Y2a & 366.1414 & 2 & 5.3 & 366.1411 & 3 & 4.4 & 366.1375 & 1 & -5.4 & 366.1416 & 3 & 5.8 \\
\hline C3Y2a & & & & & & & & & & 384.1507 & 0.2 & 1.7 \\
\hline B3Y2aY2b & 454.1570 & 1 & 3.3 & 454.1577 & 3 & 4.8 & 454.1561 & 3 & 1.3 & 454.1568 & 4 & 2.9 \\
\hline B4Y2a & 528.1937 & 3 & 2.7 & 528.1911 & 5 & -2.3 & 528.1937 & 5 & 2.7 & 528.1940 & 4 & 3.2 \\
\hline $\mathrm{B} 2 \mathrm{a}-\mathrm{H}_{2} \mathrm{O}$ & 565.1885 & 4 & 1.7 & 565.1889 & 2 & 2.4 & 565.1867 & 1 & -1.5 & 565.1868 & 2 & -1.3 \\
\hline B2a & 583.1994 & 7 & 2.2 & 583.2008 & 12 & 4.6 & 583.1980 & 12 & -0.2 & 583.1980 & 11 & -0.2 \\
\hline $\mathrm{C} 2 \mathrm{a}$ & 601.2050 & 0.2 & -6.1 & 601.2168 & 0.1 & 13.5 & 601.2086 & 0.7 & -0.1 & 601.2141 & 0.4 & 9.0 \\
\hline B4Y3aY2b & 616.2084 & 0.6 & 0.2 & 616.2189 & 0.2 & 17.2 & 616.2103 & 1 & 3.3 & 616.2118 & 0.7 & 5.7 \\
\hline B3Z3a & 639.2252 & 0.1 & 1.4 & 639.2285 & 0.3 & 6.5 & & & & 639.2238 & 0.2 & -0.8 \\
\hline В3YЗа & 657.2335 & 0.8 & -2.1 & 657.2347 & 2 & -0.3 & 657.2315 & 0.9 & -5.2 & 657.2342 & 2 & -1.0 \\
\hline $\mathrm{B} 3 \mathrm{Z2} \mathrm{b}-\mathrm{H}_{2} \mathrm{O}$ & 709.2265 & 0.2 & -4.7 & 709.2371 & 0.8 & 10.3 & 709.2331 & 0.5 & 4.7 & 709.2399 & 0.1 & 14.2 \\
\hline B3Z2b & 727.2422 & 4 & 2.5 & 727.2353 & 3 & -7.0 & 727.2433 & 1 & 4.0 & 727.2404 & 2 & 0.0 \\
\hline B3Y2b & 745.2510 & 6 & 0.1 & 745.2509 & 10 & 0.0 & 745.2500 & 11 & -1.3 & 745.2491 & 10 & -2.5 \\
\hline C3Y2b & 763.2638 & 0.1 & 3.0 & 763.2686 & 0.5 & 9.3 & 763.2576 & 0.2 & -5.1 & 763.2684 & 0.3 & 9.0 \\
\hline B4Z3a & & & & & & & 801.2788 & 0.2 & 2.1 & & & \\
\hline B4Y3a & 819.2870 & 2 & -0.9 & 819.2889 & 1 & 1.5 & 819.2876 & 4 & -0.1 & 819.2850 & 3 & -3.3 \\
\hline $\mathrm{B} 4 \mathrm{Z} 1 \mathrm{~b}-\mathrm{H}_{2} \mathrm{O}$ & 871.2805 & 0.1 & -2.4 & & & & & & & & & \\
\hline B4Z1b & 889.2930 & 1 & -0.2 & 889.2850 & 0.7 & -9.2 & 889.2911 & 0.4 & -2.4 & 889.2816 & 0.2 & -13.0 \\
\hline B4Y1b & 907.3040 & 4 & 0.2 & 907.2977 & 5 & -6.7 & 907.3011 & 7 & -3.0 & 907.3018 & 6 & -2.2 \\
\hline $\mathrm{B} 3-\mathrm{H}_{2} \mathrm{O}$ & 930.3169 & 3 & -3.0 & 930.3179 & 2 & -2.0 & 930.3217 & 1 & 2.1 & 930.3157 & 0.8 & -4.3 \\
\hline B3 & 948.3285 & 7 & -1.9 & 948.3249 & 11 & -5.7 & 948.3273 & 12 & -3.2 & 948.3286 & 9 & -1.8 \\
\hline C3 & 966.3417 & 0.8 & 0.9 & 966.3289 & 1 & -12.4 & 966.3395 & 1 & -1.4 & 966.3368 & 0.8 & -4.2 \\
\hline $\mathrm{B} 4-\mathrm{H}_{2} \mathrm{O}$ & 1092.3699 & 10 & -2.4 & 1092.3696 & 5 & -2.7 & 1092.3657 & 3 & -6.3 & 1092.3677 & 3 & -4.4 \\
\hline B4 & 1110.3809 & 28 & -2.0 & 1110.3790 & 39 & -3.7 & 1110.3784 & 51 & -4.2 & 1110.3806 & 33 & -2.3 \\
\hline C4 & & & & & & & 1128.3891 & 0.1 & -4.1 & & & \\
\hline
\end{tabular}


Table S5: (continued)

\begin{tabular}{|c|c|c|c|c|c|c|c|c|c|c|c|c|}
\hline \multirow{2}{*}{$\begin{array}{l}\text { Fragment } \\
\text { Assignment }\end{array}$} & \multicolumn{3}{|c|}{ GD2 (36:1) } & \multicolumn{3}{|c|}{ GD2 (34:1) } & \multicolumn{3}{|c|}{ GD2 (36:2) } & \multicolumn{3}{|c|}{ GD2 (36:0) } \\
\hline & $m / z$ & $1 \%$ & ppm & $m / z$ & $1 \%$ & ppm & $m / z$ & $1 \%$ & ppm & $m / z$ & $1 \%$ & ppm \\
\hline \multicolumn{13}{|c|}{ Glycolipid fragments } \\
\hline $\mathrm{ZO}-\mathrm{H}_{2} \mathrm{O}$ & & & & 502.4935 & 0.3 & -9.4 & & & & & & \\
\hline Z0 & 548.5412 & 2 & 2.0 & 520.5164 & 1 & 14.6 & 546.5226 & 3 & -3.4 & 550.5618 & 0.7 & 11.0 \\
\hline YO & 566.5510 & 0.9 & 0.5 & 538.5172 & 0.7 & -4.1 & 564.5328 & 2 & -4.0 & 568.5673 & 5 & 1.7 \\
\hline $\mathrm{Z1}$ & 710.5912 & 0.9 & -2.5 & 682.5613 & 2 & -0.6 & 708.5748 & 3 & -3.6 & & & \\
\hline Y1 & 728.6060 & 2 & 3.3 & 700.5720 & 1 & -0.4 & 726.5925 & 3 & 6.3 & 730.6184 & 3 & -1.1 \\
\hline Y2aZ2b & 872.6433 & 1 & -2.9 & 844.6162 & 0.4 & 2.0 & 870.6283 & 1 & -2.2 & 874.6575 & 0.1 & -4.5 \\
\hline Y2aY2b & 890.6545 & 4 & -2.1 & 862.6226 & 6 & -2.9 & 888.6392 & 6 & -1.7 & 892.6718 & 9 & -0.3 \\
\hline Z2a & 1075.7197 & 1 & -5.1 & 1047.6946 & 3 & 0.7 & 1073.7043 & 3 & -4.9 & 1077.7290 & 0.2 & 11.0 \\
\hline Y2a & 1093.7333 & 4 & -2.2 & 1065.6986 & 5 & -5.5 & 1091.7177 & 5 & -2.2 & 1095.7471 & 5 & -3.9 \\
\hline Z3aZ2b & & & & & & & 1143.7151 & 0.1 & 0.1 & & & \\
\hline Y3aZ2b & 1163.7465 & 0.2 & 4.5 & 1135.7113 & 0.9 & 1.2 & 1161.7314 & 0.1 & 5.0 & 1165.7423 & 0.2 & 12.5 \\
\hline Y3aY2b & 1181.7511 & 1 & -0.6 & 1153.7130 & 1 & -6.5 & 1179.7282 & 0.7 & -6.7 & 1183.7597 & 2 & -6.6 \\
\hline Z3a & 1366.8149 & 0.3 & -4.2 & 1338.7720 & 1 & -12.9 & 1364.7929 & 0.2 & -8.8 & 1368.8261 & 0.1 & -7.4 \\
\hline Y3a & 1384.8246 & 0.7 & -4.7 & 1356.8003 & 1 & 0.3 & 1382.8020 & 0.8 & -9.8 & 1386.8457 & 0.7 & -0.8 \\
\hline $\mathrm{Z} 2 \mathrm{~b}-\mathrm{H}_{2} \mathrm{O}$ & 1436.8183 & 0.7 & -5.4 & & & & & & & 1438.8166 & 0.2 & 17.5 \\
\hline $\mathrm{Z} 2 \mathrm{~b}$ & 1454.8303 & 4 & -4.4 & 1426.7925 & 4 & -9.0 & 1452.8156 & 3 & -3.7 & 1456.8493 & 2 & -2.1 \\
\hline Y2b & 1472.8400 & 12 & -4.9 & 1444.8061 & 20 & -6.8 & 1470.8189 & 19 & -8.6 & 1474.8549 & 16 & -5.4 \\
\hline $\mathrm{MH}-\mathrm{H}_{2} \mathrm{O}$ & 1657.9081 & 51 & -4.8 & 1629.8756 & 27 & -5.6 & 1655.8910 & 23 & -5.7 & 1659.9233 & 9 & -5.0 \\
\hline $\mathrm{MH}$ & 1675.9192 & 100 & -4.4 & 1647.8831 & 100 & -7.4 & 1673.8994 & 100 & -6.9 & 1677.9319 & 100 & -6.2 \\
\hline
\end{tabular}


Table S6: Table S5 data of product ion masses and fragment assignments for GD2 lipid forms GD2 (34:1), GD2 (36:0), and GD2 (36:2) presented according to the relative ion intensity (I\%) from the highest to the lowest for the carbohydrate and glycolipid fragments, respectively. The dashed line represents a $2 \%$ threshold [according to the reference, GD2 (36:1)], below which fragment matching is not always guaranteed.

\begin{tabular}{|c|c|c|c|c|c|c|c|c|}
\hline \multirow{2}{*}{$\begin{array}{l}\text { Fragment } \\
\text { Assignment }\end{array}$} & \multicolumn{2}{|c|}{ GD2 (36:1) } & \multicolumn{2}{|c|}{ GD2 (34:1) } & \multicolumn{2}{|c|}{ GD2 (36:2) } & \multicolumn{2}{|c|}{ GD2 (36:0) } \\
\hline & $m / z$ & $1 \%$ & $m / z$ & $1 \%$ & $m / z$ & $1 \%$ & $m / z$ & $1 \%$ \\
\hline \multicolumn{9}{|c|}{ Carbohydrate fragments } \\
\hline B4 & 1110.3809 & 28 & 1110.3790 & 39 & 1110.3784 & 51 & 1110.3806 & 33 \\
\hline $\mathrm{B} 4-\mathrm{H}_{2} \mathrm{O}$ & 1092.3699 & 10 & 1092.3696 & 5 & 1092.3657 & 3 & 1092.3677 & 3 \\
\hline B2a & 583.1994 & 7 & 583.2008 & 12 & 583.1980 & 12 & 583.1980 & 11 \\
\hline B3 & 948.3285 & 7 & 948.3249 & 11 & 948.3273 & 12 & 948.3286 & 9 \\
\hline B3Y2b & 745.251 & 6 & 745.2509 & 10 & 745.2500 & 11 & 745.2491 & 10 \\
\hline $\mathrm{B} 2 \mathrm{a}-\mathrm{H}_{2} \mathrm{O}$ & 565.1885 & 4 & 565.1889 & 2 & 565.1867 & 1 & 565.1868 & 2 \\
\hline B3Z2b & 727.2422 & 4 & 727.2353 & 3 & 727.2433 & 1 & 727.2404 & 2 \\
\hline B4Y1b & 907.3040 & 4 & 907.2977 & 5 & 907.3011 & 7 & 907.3018 & 6 \\
\hline B1a & 292.1059 & 3 & 292.1048 & 5 & 292.1054 & 6 & 292.1040 & 3 \\
\hline B4Y2a & 528.1937 & 3 & 528.1911 & 5 & 528.1937 & 5 & 528.1940 & 4 \\
\hline $\mathrm{B} 3-\mathrm{H}_{2} \mathrm{O}$ & 930.3169 & 3 & 930.3179 & 2 & 930.3217 & 1 & 930.3157 & 0.8 \\
\hline B2aZ3a & 274.0950 & 2 & 274.0934 & 3 & 274.0930 & 3 & 274.0946 & 3 \\
\hline B3Y2a & 366.1414 & 2 & 366.1411 & 3 & 366.1375 & 1 & 366.1416 & 3 \\
\hline B4Y3a & 819.2870 & 2 & 819.2889 & 1 & 819.2876 & 4 & 819.2850 & 3 \\
\hline $\mathrm{B}_{3} \mathrm{Y}_{2} \mathrm{aY}{ }_{2} \mathrm{~b}$ & 454.1570 & 1 & 454.1577 & 3 & 454.1561 & 3 & 454.1568 & 4 \\
\hline B4Z1b & 889.2930 & 1 & 889.2850 & 0.7 & 889.2911 & 0.4 & 889.2816 & 0.2 \\
\hline В3YЗа & 657.2335 & 0.8 & 657.2347 & 2 & 657.2315 & 0.9 & 657.2342 & 2 \\
\hline C3 & 966.3417 & 0.8 & 966.3289 & 1 & 966.3395 & 1 & 966.3368 & 0.8 \\
\hline $\mathrm{B}_{4} \mathrm{Y}_{3} \mathrm{aY} \mathrm{Y}_{2} \mathrm{~b}$ & 616.2084 & 0.6 & 616.2189 & 0.2 & 616.2103 & 1 & 616.2118 & 0.7 \\
\hline $\mathrm{C} 2 \mathrm{a}$ & 601.2050 & 0.2 & 601.2168 & 0.1 & 601.2086 & 0.7 & 601.2141 & 0.4 \\
\hline $\mathrm{B} 3 \mathrm{Z} \mathrm{b}-\mathrm{H}_{2} \mathrm{O}$ & 709.2265 & 0.2 & 709.2371 & 0.8 & 709.2331 & 0.5 & 709.2399 & 0.1 \\
\hline B3Z3a & 639.2252 & 0.1 & 639.2285 & 0.3 & & & 639.2238 & 0.2 \\
\hline C3Y2b & 763.2638 & 0.1 & 763.2686 & 0.5 & 763.2576 & 0.2 & 763.2684 & 0.3 \\
\hline $\mathrm{B} 4 \mathrm{Z} 1 \mathrm{~b}-\mathrm{H}_{2} \mathrm{O}$ & 871.2805 & 0.1 & & & & & & \\
\hline $\mathrm{C} 1 \mathrm{a}$ & & & 310.1143 & 0.3 & & & 310.1162 & 0.2 \\
\hline C3Y2a & & & & & & & 384.1507 & 0.2 \\
\hline B4Z3a & & & & & 801.2788 & 0.2 & & \\
\hline C4 & & & & & 1128.3891 & 0.1 & & \\
\hline
\end{tabular}


Table S6: (continued)

\begin{tabular}{|c|c|c|c|c|c|c|c|c|}
\hline \multirow{2}{*}{$\begin{array}{l}\text { Fragment } \\
\text { Assignment }\end{array}$} & \multicolumn{2}{|c|}{ GD2 (36:1) } & \multicolumn{2}{|c|}{ GD2 (34:1) } & \multicolumn{2}{|c|}{ GD2 (36:2) } & \multicolumn{2}{|c|}{ GD2 (36:0) } \\
\hline & $m / z$ & $1 \%$ & $m / z$ & $1 \%$ & $m / z$ & $1 \%$ & $m / z$ & $1 \%$ \\
\hline \multicolumn{9}{|c|}{ Glycolipid fragments } \\
\hline $\mathrm{MH}-\mathrm{H}_{2} \mathrm{O}$ & 1657.9081 & 51 & 1629.8756 & 27 & 1655.8910 & 23 & 1659.9233 & 9 \\
\hline Y2b & 1472.8400 & 12 & 1444.8061 & 20 & 1470.8189 & 19 & 1474.8549 & 16 \\
\hline Y2aY2b & 890.6545 & 4 & 862.6226 & 6 & 888.6392 & 6 & 892.6718 & 9 \\
\hline Y2a & 1093.7333 & 4 & 1065.6986 & 5 & 1091.7177 & 5 & 1095.7471 & 5 \\
\hline Z2b & 1454.8303 & 4 & 1426.7925 & 4 & 1452.8156 & 3 & 1456.8493 & 2 \\
\hline Z0 & 548.5412 & 2 & 520.5164 & 1 & 546.5226 & 3 & 550.5618 & 0.7 \\
\hline Y1 & 728.6060 & 2 & 700.5720 & 1 & 726.5925 & 3 & 730.6184 & 3 \\
\hline Y2aZ2b & 872.6433 & 1 & 844.6162 & 0.4 & 870.6283 & 1 & 874.6575 & 0.1 \\
\hline Z2a & 1075.7197 & 1 & 1047.6946 & 3 & 1073.7043 & 3 & 1077.7290 & 0.2 \\
\hline Y3aY2b & 1181.7511 & 1 & 1153.7130 & 1 & 1179.7282 & 0.7 & 1183.7597 & 2 \\
\hline Y0 & 566.5510 & 0.9 & 538.5172 & 0.7 & 564.5328 & 2 & 568.5673 & 5 \\
\hline Z1 & 710.5912 & 0.9 & 682.5613 & 2 & 708.5748 & 3 & & \\
\hline Y3a & 1384.8246 & 0.7 & 1356.8003 & 1 & 1382.8020 & 0.8 & 1386.8457 & 0.7 \\
\hline $\mathrm{Z} 2 \mathrm{~b}-\mathrm{H}_{2} \mathrm{O}$ & 1436.8183 & 0.7 & & & & & 1438.8166 & 0.2 \\
\hline Z3a & 1366.8149 & 0.3 & 1338.7720 & 1 & 1364.7929 & 0.2 & 1368.8261 & 0.1 \\
\hline Y3aZ2b & 1163.7465 & 0.2 & 1135.7113 & 0.9 & 1161.7314 & 0.1 & 1165.7423 & 0.2 \\
\hline $\mathrm{ZO}-\mathrm{H}_{2} \mathrm{O}$ & & & 502.4935 & 0.3 & & & & \\
\hline Z3aZ2b & & & & & 1143.7151 & 0.1 & & \\
\hline
\end{tabular}


Table S7: Product ion masses and fragment assignments for the GD2 lipid forms presented in Figure 6 and Figure S1. Due to the number of lipid forms detected, only the carbon-38 lipid forms of GD2 are given here (left to right): GD2 (36:1) (reference product ion list), GD2 (38:0), GD2 (38:1), and GD2 (38:2). For each lipid form, the observed mass $(\mathrm{m} / \mathrm{z})$, relative ion intensity $(1 \%)$, and mass accuracy (compared to the theoretically calculated ion mass) (ppm) are provided. The fragment masses have been divided into carbohydrate (top) and glycolipid fragments in order of increasing mass.

\begin{tabular}{|c|c|c|c|c|c|c|c|c|c|c|c|c|}
\hline \multirow{2}{*}{$\begin{array}{l}\text { Fragment } \\
\text { Assignment }\end{array}$} & \multicolumn{3}{|c|}{ GD2 (36:1) } & \multicolumn{3}{|c|}{ GD2 (38:2) } & \multicolumn{3}{|c|}{ GD2 (38:1) } & \multicolumn{3}{|c|}{ GD2 (38:0) } \\
\hline & $m / z$ & $1 \%$ & ppm & $m / z$ & $1 \%$ & ppm & $m / z$ & $1 \%$ & ppm & $m / z$ & $1 \%$ & ppm \\
\hline \multicolumn{13}{|c|}{ Carbohydrate fragments } \\
\hline B2aZ3a & 274.0950 & 2 & 10.5 & 274.0937 & 4 & 5.7 & 274.0942 & 0.8 & 7.6 & 274.0941 & 2 & 7.2 \\
\hline B1a & 292.1059 & 3 & 11.0 & 292.1040 & 4 & 4.5 & 292.1049 & 2 & 7.6 & 292.1056 & 3 & 10.0 \\
\hline C1a & & & & 310.1143 & 0.3 & 3.4 & & & & & & \\
\hline B3Y2a & 366.1414 & 2 & 5.3 & 366.1425 & 3 & 8.3 & 366.1421 & 2 & 7.2 & 366.1408 & 2 & 3.6 \\
\hline B3Y2aY2b & 454.1570 & 1 & 3.3 & 454.1589 & 2 & 7.5 & 454.1572 & 2 & 3.7 & 454.1579 & 3 & 5.3 \\
\hline B4Y2a & 528.1937 & 3 & 2.7 & 528.1922 & 5 & -0.2 & 528.1941 & 2 & 3.4 & 528.1943 & 3 & 3.8 \\
\hline $\mathrm{B} 2 \mathrm{a}-\mathrm{H}_{2} \mathrm{O}$ & 565.1885 & 4 & 1.7 & 565.1845 & 1 & -5.4 & 565.1886 & 3 & 1.9 & 565.1883 & 5 & 1.3 \\
\hline B2a & 583.1994 & 7 & 2.2 & 583.1983 & 14 & 0.3 & 583.1992 & 6 & 1.9 & 583.1988 & 11 & 1.2 \\
\hline $\mathrm{C} 2 \mathrm{a}$ & 601.2050 & 0.2 & -6.1 & 601.2132 & 0.1 & 7.5 & 601.2104 & 0.1 & 2.9 & 601.2140 & 0.6 & 8.9 \\
\hline B4Y3aY2b & 616.2084 & 0.6 & 0.2 & 616.2136 & 1 & 8.6 & 616.2085 & 0.5 & 0.3 & 616.2052 & 0.9 & -5.0 \\
\hline B3Z3a & 639.2252 & 0.1 & 1.4 & & & & & & & 639.2296 & 0.1 & 8.3 \\
\hline ВЗҮЗа & 657.2335 & 0.8 & -2.1 & 657.2310 & 1 & -5.9 & 657.2348 & 1 & -0.1 & 657.2292 & 0.9 & -8.7 \\
\hline $\mathrm{B} 3 \mathrm{Z} 2 \mathrm{~b}-\mathrm{H}_{2} \mathrm{O}$ & 709.2265 & 0.2 & -4.7 & 709.2294 & 0.9 & -0.6 & 709.2281 & 0.1 & -2.4 & 709.2289 & 0.3 & -1.3 \\
\hline B3Z2b & 727.2422 & 4 & 2.5 & 727.2354 & 2 & -6.8 & 727.2425 & 3 & 2.9 & 727.2428 & 5 & 3.3 \\
\hline B3Y2b & 745.2510 & 6 & 0.1 & 745.2505 & 10 & -0.6 & 745.2508 & 5 & -0.2 & 745.2506 & 10 & -0.4 \\
\hline С3Y2b & 763.2638 & 0.1 & 3.0 & 763.2728 & 0.2 & 14.8 & 763.2548 & 0.1 & -8.8 & 763.2624 & 0.2 & 1.2 \\
\hline B4Z3a & & & & 801.2750 & 0.5 & -2.6 & & & & & & \\
\hline В4Y3a & 819.2870 & 2 & -0.9 & 819.2866 & 3 & -1.3 & 819.2877 & 2 & 0.0 & 819.2857 & 3 & -2.4 \\
\hline $\mathrm{B} 4 \mathrm{Z} 1 \mathrm{~b}-\mathrm{H}_{2} \mathrm{O}$ & 871.2805 & 0.1 & -2.4 & & & & 871.2785 & 0.1 & -4.7 & 871.2810 & 0.1 & -1.9 \\
\hline B4Z1b & 889.2930 & 1 & -0.2 & 889.2902 & 0.6 & -3.4 & 889.2958 & 1 & 2.9 & 889.2925 & 1 & -0.8 \\
\hline B4Y1b & 907.3040 & 4 & 0.2 & 907.3024 & 7 & -1.5 & 907.3039 & 3 & 0.1 & 907.3026 & 5 & -1.3 \\
\hline B3- $\mathrm{H}_{2} \mathrm{O}$ & 930.3169 & 3 & -3.0 & 930.3152 & 0.9 & -4.9 & 930.3191 & 3 & -0.7 & 930.3187 & 3 & -1.1 \\
\hline B3 & 948.3285 & 7 & -1.9 & 948.3294 & 10 & -1.0 & 948.3285 & 7 & -1.9 & 948.3294 & 10 & -1.0 \\
\hline C3 & 966.3417 & 0.8 & 0.9 & 966.3429 & 2 & 2.1 & 966.3398 & 0.5 & -1.1 & 966.3434 & 1 & 2.6 \\
\hline $\mathrm{B} 4-\mathrm{H}_{2} \mathrm{O}$ & 1092.3699 & 10 & -2.4 & 1092.3683 & 3 & -3.9 & 1092.3701 & 10 & -2.2 & 1092.3688 & 11 & -3.4 \\
\hline B4 & 1110.3809 & 28 & -2.0 & 1110.3790 & 40 & -3.7 & 1110.3795 & 28 & -3.2 & 1110.3813 & 29 & -1.6 \\
\hline
\end{tabular}


Table S7: (continued)

\begin{tabular}{|c|c|c|c|c|c|c|c|c|c|c|c|c|}
\hline \multirow{2}{*}{$\begin{array}{l}\text { Fragment } \\
\text { Assignment }\end{array}$} & \multicolumn{3}{|c|}{ GD2 (36:1) } & \multicolumn{3}{|c|}{ GD2 (38:2) } & \multicolumn{3}{|c|}{ GD2 (38:1) } & \multicolumn{3}{|c|}{ GD2 (38:0) } \\
\hline & $m / z$ & $1 \%$ & ppm & $m / z$ & $1 \%$ & ppm & $m / z$ & $1 \%$ & ppm & $m / z$ & $1 \%$ & ppm \\
\hline \multicolumn{13}{|c|}{ Glycolipid fragments } \\
\hline Z0 & 548.5412 & 2 & 2.0 & 574.5563 & 2 & 1.0 & 576.5737 & 2 & 4.0 & 578.5971 & 0.6 & 17.4 \\
\hline Y0 & 566.5510 & 0.9 & 0.5 & 592.5661 & 1 & -0.4 & 594.5825 & 0.9 & 0.8 & 596.6000 & 4 & 3.9 \\
\hline $\mathrm{Z1}$ & 710.5912 & 0.9 & -2.5 & 736.6139 & 0.8 & 7.1 & 738.6231 & 1 & -1.6 & & & \\
\hline Y1 & 728.6060 & 2 & 3.3 & 754.6218 & 1 & 3.4 & 756.6345 & 2 & -0.5 & 758.6491 & 3 & -1.9 \\
\hline Y2aZ2b & 872.6433 & 1 & -2.9 & 898.6564 & 0.4 & -5.6 & 900.6762 & 1 & -1.0 & & & \\
\hline Y2aY2b & 890.6545 & 4 & -2.1 & 916.6672 & 2 & -5.3 & 918.6854 & 3 & -2.5 & 920.7015 & 6 & -2.0 \\
\hline $\mathrm{Z2a}$ & 1075.7197 & 1 & -5.1 & 1101.7331 & 1 & -7.0 & 1103.7546 & 2 & -1.7 & & & \\
\hline Y2a & 1093.7333 & 4 & -2.2 & 1119.7527 & 3 & 1.2 & 1121.7629 & 3 & -3.7 & 1123.7779 & 6 & -4.3 \\
\hline Y3aZ2b & 1163.7465 & 0.2 & 4.5 & 1189.7388 & 0.5 & -15.2 & 1191.7703 & 0.4 & -1.9 & 1193.7959 & 0.1 & 6.5 \\
\hline Y3aY2b & 1181.7511 & 1 & -0.6 & 1207.7767 & 1 & 7.7 & 1209.7822 & 0.7 & -0.7 & 1211.7923 & 1 & -5.3 \\
\hline Z3a & 1366.8149 & 0.3 & -4.2 & & & & 1394.8533 & 0.1 & 1.0 & 1396.8888 & 0.1 & 15.2 \\
\hline Y3a & 1384.8246 & 0.7 & -4.7 & 1410.8215 & 1 & -18.0 & 1412.8572 & 0.8 & -3.7 & 1414.8628 & 0.9 & -10.8 \\
\hline $\mathrm{Z} 2 \mathrm{~b}-\mathrm{H}_{2} \mathrm{O}$ & 1436.8183 & 0.7 & -5.4 & 1462.8407 & 0.4 & -0.7 & 1464.8503 & 0.5 & -4.8 & 1466.8671 & 0.04 & -4.0 \\
\hline $\mathrm{Z} 2 \mathrm{~b}$ & 1454.8303 & 4 & -4.4 & 1480.8372 & 2 & -10.2 & 1482.8614 & 4 & -4.4 & 1484.8763 & 3 & -4.9 \\
\hline Y2b & 1472.8400 & 12 & -4.9 & 1498.8531 & 10 & -6.5 & 1500.8733 & 11 & -3.5 & 1502.8842 & 11 & -6.6 \\
\hline $\mathrm{MH}-\mathrm{H}_{2} \mathrm{O}$ & 1657.9081 & 51 & -4.8 & 1683.9297 & 17 & -1.2 & 1685.9389 & 51 & -5.0 & 1687.9521 & 27 & -6.4 \\
\hline $\mathrm{MH}$ & 1675.9192 & 100 & -4.4 & 1701.9307 & 100 & -6.8 & 1703.9492 & 100 & -5.1 & 1705.9631 & 100 & -6.1 \\
\hline
\end{tabular}


Table S8: Table S7 data of product ion masses and fragment assignments for GD2 lipid forms GD2 (36:1), GD2 (38:0), GD2 (38:1), and GD2 (38:2) presented according to the relative ion intensity (1\%) from the highest to the lowest for the carbohydrate and glycolipid fragments, respectively. The dashed line represents a $2 \%$ threshold (according to the reference, GD2 (36:1)), below which fragment matching is not always guaranteed.

\begin{tabular}{|c|c|c|c|c|c|c|c|c|}
\hline \multirow{2}{*}{$\begin{array}{l}\text { Fragment } \\
\text { Assignment }\end{array}$} & \multicolumn{2}{|c|}{ GD2 (36:1) } & \multicolumn{2}{|c|}{ GD2 (38:2) } & \multicolumn{2}{|c|}{ GD2 (38:1) } & \multicolumn{2}{|c|}{ GD2 (38:0) } \\
\hline & $m / z$ & $1 \%$ & $m / z$ & $1 \%$ & $m / z$ & $1 \%$ & $m / z$ & $1 \%$ \\
\hline \multicolumn{9}{|c|}{ Carbohydrate fragments } \\
\hline B2a & 583.1994 & 7 & 583.1983 & 14 & 583.1992 & 6 & 583.1988 & 11 \\
\hline B3Y2b & 745.2510 & 6 & 745.2505 & 10 & 745.2508 & 5 & 745.2506 & 10 \\
\hline $\mathrm{B} 2 \mathrm{a}-\mathrm{H}_{2} \mathrm{O}$ & 565.1885 & 4 & 565.1845 & 1 & 565.1886 & 3 & 565.1883 & 5 \\
\hline B3Z2b & 727.2422 & 4 & 727.2354 & 2 & 727.2425 & 3 & 727.2428 & 5 \\
\hline B1a & 292.1059 & 3 & 292.1040 & 4 & 292.1049 & 2 & 292.1056 & 3 \\
\hline B4Y2a & 528.1937 & 3 & 528.1922 & 5 & 528.1941 & 2 & 528.1943 & 3 \\
\hline B2aZ3a & 274.0950 & 2 & 274.0937 & 4 & 274.0942 & 0.8 & 274.0941 & 2 \\
\hline B3Y2a & 366.1414 & 2 & 366.1425 & 3 & 366.1421 & 2 & 366.1408 & 2 \\
\hline B4Y3a & 819.2870 & 2 & 819.2866 & 3 & 819.2877 & 2 & 819.2857 & 3 \\
\hline B3Y2aY2b & 454.1570 & 1 & 454.1589 & 2 & 454.1572 & 2 & 454.1579 & 3 \\
\hline B4Z1b & 889.2930 & 1 & 889.2902 & 0.6 & 889.2958 & 1 & 889.2925 & 1 \\
\hline В3YЗа & 657.2335 & 0.8 & 657.2310 & 1 & 657.2348 & 1 & 657.2292 & 0.9 \\
\hline B4Y3aY2b & 616.2084 & 0.6 & 616.2136 & 1 & 616.2085 & 0.5 & 616.2052 & 0.9 \\
\hline $\mathrm{C} 2 \mathrm{a}$ & 601.2050 & 0.2 & 601.2132 & 0.1 & 601.2104 & 0.1 & 601.2140 & 0.6 \\
\hline $\mathrm{B} 3 \mathrm{Z2b}-\mathrm{H}_{2} \mathrm{O}$ & 709.2265 & 0.2 & 709.2294 & 0.9 & 709.2281 & 0.1 & 709.2289 & 0.3 \\
\hline B3Z3a & 639.2252 & 0.1 & & & & & 639.2296 & 0.1 \\
\hline C3Y2b & 763.2638 & 0.1 & 763.2728 & 0.2 & 763.2548 & 0.1 & 763.2624 & 0.2 \\
\hline $\mathrm{B} 4 \mathrm{Z1b}-\mathrm{H}_{2} \mathrm{O}$ & 871.2805 & 0.1 & & & 871.2785 & 0.1 & 871.2810 & 0.1 \\
\hline $\mathrm{C} 1 \mathrm{a}$ & & & 310.1143 & 0.3 & & & & \\
\hline B4Z3a & & & 801.2750 & 0.5 & & & & \\
\hline
\end{tabular}


Table S8: (continued)

\begin{tabular}{|c|c|c|c|c|c|c|c|c|}
\hline \multirow{2}{*}{$\begin{array}{l}\text { Fragment } \\
\text { Assignment }\end{array}$} & \multicolumn{2}{|c|}{ GD2 (36:1) } & \multicolumn{2}{|c|}{ GD2 (38:2) } & \multicolumn{2}{|c|}{ GD2 (38:1) } & \multicolumn{2}{|c|}{ GD2 (38:1) } \\
\hline & $m / z$ & $1 \%$ & $m / z$ & $1 \%$ & $m / z$ & $1 \%$ & $m / z$ & $1 \%$ \\
\hline \multicolumn{9}{|c|}{ Glycolipid fragments } \\
\hline $\mathrm{MH}-\mathrm{H}_{2} \mathrm{O}$ & 1657.9081 & 51 & 1683.9297 & 17 & 1685.9389 & 51 & 1687.9521 & 27 \\
\hline Y2b & 1472.8400 & 12 & 1498.8531 & 10 & 1500.8733 & 11 & 1502.8842 & 11 \\
\hline Y2aY2b & 890.6545 & 4 & 916.6672 & 2 & 918.6854 & 3 & 920.7015 & 6 \\
\hline Y2a & 1093.7333 & 4 & 1119.7527 & 3 & 1121.7629 & 3 & 1123.7779 & 6 \\
\hline $\mathrm{Z} 2 \mathrm{~b}$ & 1454.8303 & 4 & 1480.8372 & 2 & 1482.8614 & 4 & 1484.8763 & 3 \\
\hline Z0 & 548.5412 & 2 & 574.5563 & 2 & 576.5737 & 2 & 578.5971 & 0.6 \\
\hline Y1 & 728.6060 & 2 & 754.6218 & 1 & 756.6345 & 2 & 758.6491 & 3 \\
\hline Y2aZ2b & 872.6433 & 1 & 898.6564 & 0.4 & 900.6762 & 1 & & \\
\hline $\mathrm{Z2a}$ & 1075.7197 & 1 & 1101.7331 & 1 & 1103.7546 & 2 & & \\
\hline Y3aY2b & 1181.7511 & 1 & 1207.7767 & 1 & 1209.7822 & 0.7 & 1211.7923 & 1 \\
\hline Y0 & 566.5510 & 0.9 & 592.5661 & 1 & 594.5825 & 0.9 & 596.6000 & 4 \\
\hline Z1 & 710.5912 & 0.9 & 736.6139 & 0.8 & 738.6231 & 1 & & \\
\hline Y3a & 1384.8246 & 0.7 & 1410.8215 & 1 & 1412.8572 & 0.8 & 1414.8628 & 0.9 \\
\hline $\mathrm{Z} 2 \mathrm{~b}-\mathrm{H}_{2} \mathrm{O}$ & 1436.8183 & 0.7 & 1462.8407 & 0.4 & 1464.8503 & 0.5 & 1466.8671 & 0.04 \\
\hline Z3a & 1366.8149 & 0.3 & & & 1394.8533 & 0.1 & 1396.8888 & 0.1 \\
\hline Y3aZ2b & 1163.7465 & 0.2 & 1189.7388 & 0.5 & 1191.7703 & 0.4 & 1193.7959 & 0.1 \\
\hline
\end{tabular}


Table S9: Product ion masses and fragment assignments for the GD2 lipid forms presented in Figure 6 and Figure S1. Due to the number of lipid forms detected, only the carbon-40 lipid forms of GD2 are given here (left to right): GD2 (36:1) (reference product ion list), GD2 (40:1), and GD2 (40:2). For each lipid form, the observed mass $(\mathrm{m} / \mathrm{z}$ ), relative ion intensity $(1 \%)$, and mass accuracy (compared to the theoretically calculated ion mass) (ppm) are provided. The fragment masses have been divided into carbohydrate (top) and glycolipid fragments in order of increasing mass.

\begin{tabular}{|c|c|c|c|c|c|c|c|c|c|}
\hline \multirow{2}{*}{$\begin{array}{l}\text { Fragment } \\
\text { Assignment }\end{array}$} & \multicolumn{3}{|c|}{ GD2 (36:1) } & \multicolumn{3}{|c|}{ GD2 (40:1) } & \multicolumn{3}{|c|}{ GD2 (40:0) } \\
\hline & $m / z$ & $1 \%$ & ppm & $m / z$ & $1 \%$ & ppm & $m / z$ & $1 \%$ & ppm \\
\hline \multicolumn{10}{|c|}{ Carbohydrate fragments } \\
\hline B2aZ3a & 274.0950 & 2 & 10.5 & 274.0923 & 2 & 0.6 & 274.0949 & 1 & 10.1 \\
\hline B1a & 292.1059 & 3 & 11.0 & 292.1049 & 3 & 7.6 & 292.1049 & 7 & 7.6 \\
\hline $\mathrm{C} 1 \mathrm{a}$ & & & & 310.1147 & 0.2 & 4.7 & 310.1165 & 0.3 & 10.5 \\
\hline B3Y2a & 366.1414 & 2 & 5.3 & 366.1407 & 2 & 3.4 & 366.1402 & 4 & 2.0 \\
\hline С3Y2a & & & & 384.1557 & 0.1 & 14.7 & & & \\
\hline B3Y2aY2b & 454.1570 & 1 & 3.3 & 454.1574 & 3 & 4.2 & 454.1632 & 2 & 17.0 \\
\hline B4Y2a & 528.1937 & 3 & 2.7 & 528.1942 & 3 & 3.6 & 528.1926 & 3 & 0.6 \\
\hline $\mathrm{B} 2 \mathrm{a}-\mathrm{H}_{2} \mathrm{O}$ & 565.1885 & 4 & 1.7 & 565.1893 & 2 & 3.1 & 565.1930 & 3 & 9.7 \\
\hline B2a & 583.1994 & 7 & 2.2 & 583.1983 & 10 & 0.3 & 583.1983 & 13 & 0.3 \\
\hline $\mathrm{C} 2 \mathrm{a}$ & 601.2050 & 0.2 & -6.1 & 601.2112 & 0.2 & 4.2 & 601.2169 & 0.3 & 13.7 \\
\hline B4Y3aY2b & 616.2084 & 0.6 & 0.2 & 616.2060 & 0.5 & -3.7 & 616.2074 & 0.2 & -1.5 \\
\hline B3Z3a & 639.2252 & 0.1 & 1.4 & & & & & & \\
\hline В3YЗа & 657.2335 & 0.8 & -2.1 & 657.2427 & 1 & 11.9 & 657.2291 & 1 & -8.8 \\
\hline $\mathrm{B} 3 \mathrm{Z} 2 \mathrm{~b}-\mathrm{H}_{2} \mathrm{O}$ & 709.2265 & 0.2 & -4.7 & 709.2318 & 0.4 & 2.8 & 709.2368 & 0.2 & 9.9 \\
\hline B3Z2b & 727.2422 & 4 & 2.5 & 727.2433 & 3 & 4.0 & 727.2448 & 2 & 6.1 \\
\hline B3Y2b & 745.2510 & 6 & 0.1 & 745.2510 & 6 & 0.1 & 745.2512 & 11 & 0.4 \\
\hline C3Y2b & 763.2638 & 0.1 & 3.0 & 763.2570 & 0.2 & -5.9 & 763.2675 & 0.4 & 7.9 \\
\hline B4Y3a & 819.2870 & 2 & -0.9 & 819.2880 & 2 & 0.4 & 819.2826 & 2 & -6.2 \\
\hline $\mathrm{B} 4 \mathrm{Z} 1 \mathrm{~b}-\mathrm{H}_{2} \mathrm{O}$ & 871.2805 & 0.1 & -2.4 & 871.2777 & 0.1 & -5.7 & & & \\
\hline B4Z1b & 889.2930 & 1 & -0.2 & 889.2949 & 1 & 1.9 & 889.2895 & 1 & -4.2 \\
\hline B4Y1b & 907.3040 & 4 & 0.2 & 907.3006 & 5 & -3.5 & 907.3041 & 6 & 0.3 \\
\hline B3- $\mathrm{H}_{2} \mathrm{O}$ & 930.3169 & 3 & -3.0 & 930.3171 & 2 & -2.8 & 930.3032 & 0.1 & -17.8 \\
\hline B3 & 948.3285 & 7 & -1.9 & 948.3288 & 9 & -1.6 & 948.3286 & 7 & -1.8 \\
\hline C3 & 966.3417 & 0.8 & 0.9 & 966.3431 & 1 & 2.3 & 966.3555 & 0.3 & 15.1 \\
\hline $\mathrm{B} 4-\mathrm{H}_{2} \mathrm{O}$ & 1092.3699 & 10 & -2.4 & 1092.3692 & 7 & -3.0 & 1092.3678 & 5 & -4.3 \\
\hline B4 & 1110.3809 & 28 & -2.0 & 1110.3816 & 37 & -1.4 & 1110.3803 & 37 & -2.5 \\
\hline
\end{tabular}


Table S9: (continued)

\begin{tabular}{|c|c|c|c|c|c|c|c|c|c|}
\hline \multirow{2}{*}{$\begin{array}{l}\text { Fragment } \\
\text { Assignment }\end{array}$} & \multicolumn{3}{|c|}{ GD2 (36:1) } & \multicolumn{3}{|c|}{ GD2 (40:1) } & \multicolumn{3}{|c|}{ GD2 (40:0) } \\
\hline & $m / z$ & $1 \%$ & ppm & $m / z$ & $1 \%$ & ppm & $m / z$ & $1 \%$ & ppm \\
\hline \multicolumn{10}{|c|}{ Glycolipid fragments } \\
\hline $\mathrm{ZO}-\mathrm{H}_{2} \mathrm{O}$ & & & & 586.6004 & 0.2 & 14.1 & & & \\
\hline Z0 & 548.5412 & 2 & 2.0 & 604.6024 & 1 & -0.5 & $606.6147^{*}$ & & -6.1 \\
\hline YO & 566.5510 & 0.9 & 0.5 & 622.6168 & 2 & 5.6 & 624.6252 & 4 & -6.0 \\
\hline $\mathrm{Z1}$ & 710.5912 & 0.9 & -2.5 & 766.6573 & 0.9 & 2.2 & 768.6576 & 0.6 & -17.8 \\
\hline Y1 & 728.6060 & 2 & 3.3 & 784.6670 & 3 & 1.1 & 786.6788 & 1 & -3.8 \\
\hline Y2aZ2b & 872.6433 & 1 & -2.9 & 928.7037 & 1 & -5.1 & & & \\
\hline Y2aY2b & 890.6545 & 4 & -2.1 & 946.7165 & 4 & -2.6 & 948.7314 & 6 & -3.4 \\
\hline Z2a & 1075.7197 & 1 & -5.1 & 1131.7830 & 2 & -4.2 & 1133.8006 & 0.5 & -2.5 \\
\hline Y2a & 1093.7333 & 4 & -2.2 & 1149.7950 & 5 & -2.9 & 1151.8067 & 6 & -6.3 \\
\hline Z3aZ2b & & & & 1201.7939 & 0.1 & 0.5 & & & \\
\hline Y3aZ2b & 1163.7465 & 0.2 & 4.5 & 1219.8073 & 0.1 & 2.8 & & & \\
\hline Y3aY2b & 1181.7511 & 1 & -0.6 & 1237.8049 & 1 & -7.7 & 1239.8209 & 2 & -7.4 \\
\hline Z3a & 1366.8149 & 0.3 & -4.2 & 1422.8832 & 0.4 & 0.0 & 1424.9067 & 0.2 & 5.5 \\
\hline Y3a & 1384.8246 & 0.7 & -4.7 & 1440.8843 & 0.5 & -6.6 & 1442.9164 & 0.4 & 4.8 \\
\hline $\mathrm{Z} 2 \mathrm{~b}-\mathrm{H}_{2} \mathrm{O}$ & 1436.8183 & 0.7 & -5.4 & 1492.8796 & 0.7 & -6.1 & & & \\
\hline Z2b & 1454.8303 & 4 & -4.4 & 1510.8915 & 3 & -5.1 & 1512.9142 & 1 & -0.5 \\
\hline Y2b & 1472.8400 & 12 & -4.9 & 1528.9040 & 11 & -3.8 & 1530.9087 & 16 & -11.0 \\
\hline $\mathrm{MH}-\mathrm{H}_{2} \mathrm{O}$ & 1657.9081 & 51 & -4.8 & 1713.9704 & 30 & -4.8 & 1715.9817 & 15 & -7.3 \\
\hline $\mathrm{MH}$ & 1675.9192 & 100 & -4.4 & 1731.9798 & 100 & -5.4 & 1733.9926 & 100 & -7.1 \\
\hline
\end{tabular}

*Isotopic pattern interference. 
Table S10: Table S9 data of product ion masses and fragment assignments for GD2 lipid forms GD2 (36:1), GD2 (40:1), and GD2 (40:2) presented according to the relative ion intensity (1\%) from the highest to the lowest for the carbohydrate and glycolipid fragments, respectively. The dashed line represents a $2 \%$ threshold (according to the reference, GD2 (36:1)), below which fragment matching is not always guaranteed.

\begin{tabular}{|c|c|c|c|c|c|c|}
\hline \multirow{2}{*}{$\begin{array}{l}\text { Fragment } \\
\text { Assignment }\end{array}$} & \multicolumn{2}{|c|}{ GD2 (36:1) } & \multicolumn{2}{|c|}{ GD2 (40:1) } & \multicolumn{2}{|c|}{ GD2 (40:0) } \\
\hline & $m / z$ & $1 \%$ & $m / z$ & $1 \%$ & $m / z$ & $1 \%$ \\
\hline \multicolumn{7}{|c|}{ Carbohydrate fragments } \\
\hline B4 & 1110.3809 & 28 & 1110.3816 & 37 & 1110.3803 & 37 \\
\hline $\mathrm{B} 4-\mathrm{H}_{2} \mathrm{O}$ & 1092.3699 & 10 & 1092.3692 & 7 & 1092.3678 & 5 \\
\hline B2a & 583.1994 & 7 & 583.1983 & 10 & 583.1983 & 13 \\
\hline B3 & 948.3285 & 7 & 948.3288 & 9 & 948.3286 & 7 \\
\hline B3Y2b & 745.2510 & 6 & 745.2510 & 6 & 745.2512 & 11 \\
\hline $\mathrm{B} 2 \mathrm{a}-\mathrm{H}_{2} \mathrm{O}$ & 565.1885 & 4 & 565.1893 & 2 & 565.1930 & 3 \\
\hline B3Z2b & 727.2422 & 4 & 727.2433 & 3 & 727.2448 & 2 \\
\hline B4Y1b & 907.3040 & 4 & 907.3006 & 5 & 907.3041 & 6 \\
\hline B1a & 292.1059 & 3 & 292.1049 & 3 & 292.1049 & 7 \\
\hline B4Y2a & 528.1937 & 3 & 528.1942 & 3 & 528.1926 & 3 \\
\hline $\mathrm{B} 3-\mathrm{H}_{2} \mathrm{O}$ & 930.3169 & 3 & 930.3171 & 2 & 930.3032 & 0.1 \\
\hline B2aZ3a & 274.0950 & 2 & 274.0923 & 2 & 274.0949 & 1 \\
\hline B3Y2a & 366.1414 & 2 & 366.1407 & 2 & 366.1402 & 4 \\
\hline В4Y3a & 819.2870 & 2 & 819.2880 & 2 & 819.2826 & 2 \\
\hline B3Y2aY2b & 454.1570 & 1 & 454.1574 & 3 & 454.1632 & 2 \\
\hline B4Z1b & 889.2930 & 1 & 889.2949 & 1 & 889.2895 & 1 \\
\hline В3ҮЗа & 657.2335 & 0.8 & 657.2427 & 1 & 657.2291 & 1 \\
\hline C3 & 966.3417 & 0.8 & 966.3431 & 1 & 966.3555 & 0.3 \\
\hline B4Y3aY2b & 616.2084 & 0.6 & 616.2060 & 0.5 & 616.2074 & 0.2 \\
\hline $\mathrm{C} 2 \mathrm{a}$ & 601.2050 & 0.2 & 601.2112 & 0.2 & 601.2169 & 0.3 \\
\hline $\mathrm{B} 3 \mathrm{Z} 2 \mathrm{~b}-\mathrm{H}_{2} \mathrm{O}$ & 709.2265 & 0.2 & 709.2318 & 0.4 & 709.2368 & 0.2 \\
\hline B3Z3a & 639.2252 & 0.1 & & & & \\
\hline C3Y2b & 763.2638 & 0.1 & 763.2570 & 0.2 & 763.2675 & 0.4 \\
\hline $\mathrm{B} 4 \mathrm{Z1} \mathrm{b}-\mathrm{H}_{2} \mathrm{O}$ & 871.2805 & 0.1 & 871.2777 & 0.1 & & \\
\hline $\mathrm{C} 1 \mathrm{a}$ & & & 310.1147 & 0.2 & 310.1165 & 0.3 \\
\hline C3Y2a & & & 384.1557 & 0.1 & & \\
\hline
\end{tabular}


Table S10: (continued)

\begin{tabular}{|c|c|c|c|c|c|c|}
\hline \multirow{2}{*}{$\begin{array}{l}\text { Fragment } \\
\text { Assignment }\end{array}$} & \multicolumn{2}{|c|}{ GD2 (36:1) } & \multicolumn{2}{|c|}{ GD2 (40:1) } & \multicolumn{2}{|c|}{ GD2 (40:0) } \\
\hline & $m / z$ & $1 \%$ & $m / z$ & $1 \%$ & $m / z$ & $1 \%$ \\
\hline \multicolumn{7}{|c|}{ Glycolipid fragments } \\
\hline $\mathrm{MH}-\mathrm{H}_{2} \mathrm{O}$ & 1657.9081 & 51 & 1713.9704 & 30 & 1715.9817 & 15 \\
\hline Y2b & 1472.8400 & 12 & 1528.9040 & 11 & 1530.9087 & 16 \\
\hline Y2aY2b & 890.6545 & 4 & 946.7165 & 4 & 948.7314 & 6 \\
\hline Y2a & 1093.7333 & 4 & 1149.7950 & 5 & 1151.8067 & 6 \\
\hline Z2b & 1454.8303 & 4 & 1510.8915 & 3 & 1512.9142 & 1 \\
\hline Z0 & 548.5412 & 2 & 604.6024 & 1 & $606.6147^{*}$ & \\
\hline Y1 & 728.6060 & 2 & 784.6670 & 3 & 786.6788 & 1 \\
\hline Y2aZ2b & 872.6433 & 1 & 928.7037 & 1 & & \\
\hline $\mathrm{Z2a}$ & 1075.7197 & 1 & 1131.7830 & 2 & 1133.8006 & 0.5 \\
\hline Y3aY2b & 1181.7511 & 1 & 1237.8049 & 1 & 1239.8209 & 2 \\
\hline YO & 566.5510 & 0.9 & 622.6168 & 2 & 624.6252 & 4 \\
\hline Z1 & 710.5912 & 0.9 & 766.6573 & 0.9 & 768.6576 & 0.6 \\
\hline ҮЗа & 1384.8246 & 0.7 & 1440.8843 & 0.5 & 1442.9164 & 0.4 \\
\hline $\mathrm{Z} 2 \mathrm{~b}-\mathrm{H}_{2} \mathrm{O}$ & 1436.8183 & 0.7 & 1492.8796 & 0.7 & & \\
\hline Z3a & 1366.8149 & 0.3 & 1422.8832 & 0.4 & 1424.9067 & 0.2 \\
\hline Y3aZ2b & 1163.7465 & 0.2 & 1219.8073 & 0.1 & & \\
\hline $\mathrm{ZO}-\mathrm{H}_{2} \mathrm{O}$ & & & 586.6004 & 0.2 & & \\
\hline Z3aZ2b & & & 1201.7939 & 0.1 & & \\
\hline
\end{tabular}

*Isotopic pattern interference. 